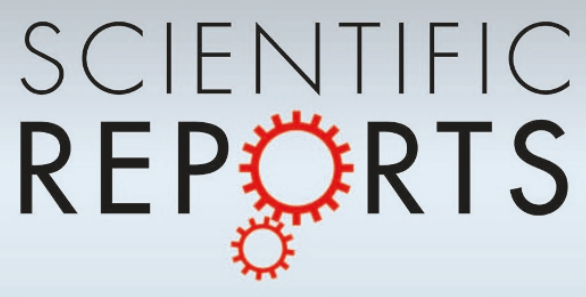

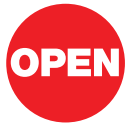

SUBJECT AREAS:

NEURODEVELOPMENTAL

DISORDERS

DEVELOPMENT

EPIGENETICS

BIOINORGANIC CHEMISTRY

Received

30 June 2011

Accepted

30 September 2011

Published

3 November 2011

Correspondence and requests for materials should be addressed to H.Y. (yasuda@lbv.co.

\section{Infantile zinc deficiency: Association with autism spectrum disorders}

\author{
Hiroshi Yasuda', Kazuya Yoshida', Yuichi Yasuda² \& Toyoharu Tsutsui ${ }^{1}$ \\ 'La Belle Vie Research Laboratory, Tokyo 103-0006, Japan, ${ }^{2}$ Health Science Laboratory, Tokyo, Japan.
}

Elucidation of the pathogenesis and effective treatment of autism spectrum disorders is one of the challenges today. In this study, we examine hair zinc concentrations for 1,967 children with autistic disorders (1,553 males and 414 females), and show considerable association with zinc deficiency. Histogram of hair zinc concentration was non-symmetric with tailing in lower range, and 584 subjects were found to have lower zinc concentrations than -2 standard deviation level of its reference range (86.3-193 ppm). The incidence rate of zinc deficiency in infant group aged 0-3 year-old was estimated $43.5 \%$ in male and $52.5 \%$ in female. The lowest zinc concentration of $10.7 \mathrm{ppm}$ was detected in a 2-year-old boy, corresponding to about $1 / 12$ of the control mean level. These findings suggest that infantile zinc deficiency may epigenetically contribute to the pathogenesis of autism and nutritional approach may yield a novel hope for its treatment and prevention.

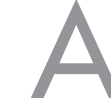

utism spectrum disorders are a group of neural development disorders characterized by impairments in reciprocal social interaction and communication, and by the presence of restricted and repetitive behaviors ${ }^{1}$, affecting 1 in 150 children in developed countries ${ }^{2}$. Therefore, the elucidation of the pathogenesis and effective treatment of autistic disorders is one of the challenges today. Autism spectrum disorders is thought to be heritable and some related genes have been reported ${ }^{3,4}$. However, the underlying genetic determinants are still unclarified ${ }^{1}$ and the interaction of heritable factors with uncertified lifestyle and environmental factors seem play a significant role in the pathogenesis. For example, ethyl-mercury, a component in vaccines has been claimed to be one of the environmental candidates causing autistic disorders ${ }^{5}$, but its relationship with autism remains to be proved scientifically. Recently, epigenetic alteration of gene expression by environmental influences is considered one of key events in the pathogenesis of genetic diseases ${ }^{6}$. And some toxic elements such as cadmium, arsenic and mercury, and also essential mineral deficiencies have been reported to be candidate factors that induce epigenetic disorders ${ }^{7-9}$.

Recent great advances in high-sensitive and reliable trace element analysis method using inductively coupled plasma mass spectrometry (ICP-MS) have enabled it to be applied for estimating chronic toxic metal burden and essential mineral deficiency in human body, showing that human blood mineral concentrations reflect to their levels in hair or nail specimen ${ }^{10,11}$. Thus, clinical metallomics studies with reliable ICP-MS method have been tried for investigating some association of several diseases and symptoms with the dynamics of trace bio-elements such as toxic metals and essential minerals ${ }^{12-18}$.

The importance of zinc in human nutrition and health has been recognized since the early 1960s. For assessment of zinc nutritional status, the use of serum zinc alone is subject to limitations because its level seems be influenced by factors other than dietary zinc intake; for example, hypoalbuminemia, infection, acute stress, pregnancy and use of oral contraceptive agents can lower serum zinc values ${ }^{19}$. In addition, serum zinc levels are subject to diurnal variation and influenced by the fasting status of the subjects.

For the last five years, we have examined on the association of toxic metal burden with autistic disorders, and reported that some parts of the children with autism have suffered from high accumulation of toxic metals such as cadmium, lead or aluminium more than mercury ${ }^{20-22}$.

In this preliminary study, we have determined human scalp hair zinc concentrations for 1,967 children diagnosed with autistic spectrum disorders by their physicians, and showed that many of them, especially in the infant group aged $0-3$ year-old, are suffering from zinc deficiency.

\section{Results}

The histogram of logarithmic hair zinc concentrations for 1,967 children diagnosed with autism by their physicians is shown in Figure 1. The distribution of the logarithmic zinc concentration was non-symmetric with 


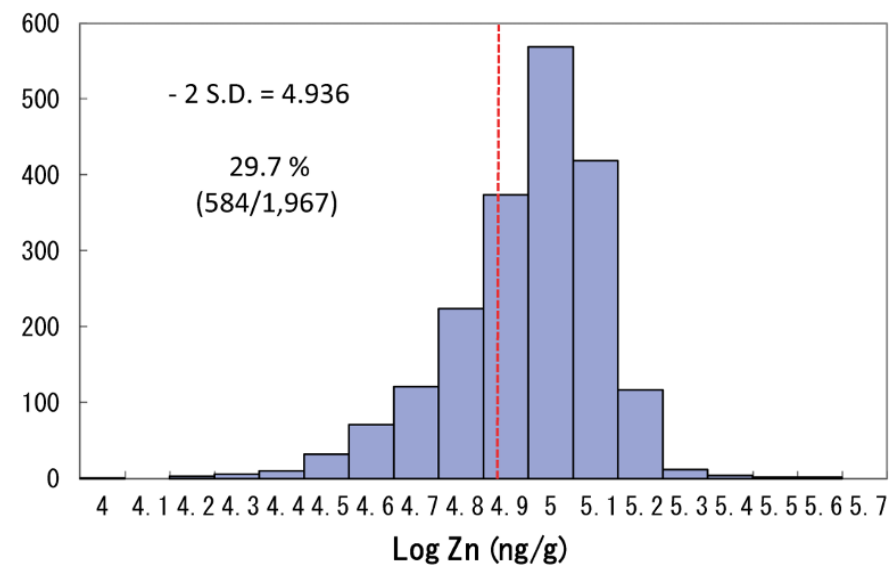

Figure $1 \mid$ Histogram of logarithmic zinc concentration in autistic children $(\mathrm{N}=1,967)$. The histogram of scalp hair zinc concentrations for 1,967 children (1,553 males and 414 females) aged $0-15$ year-old is shown in the logarithm. The numbers on the abscissa indicate the logarithms of scalp hair zinc concentrations (ng/g hair: ppb). The height of each rectangle represents the frequency in the class interval in logarithmic hair zinc level. The dotted vertical line represents the $-2 S$.D. (standard deviation) level of the control reference range of hair zinc concentrations.

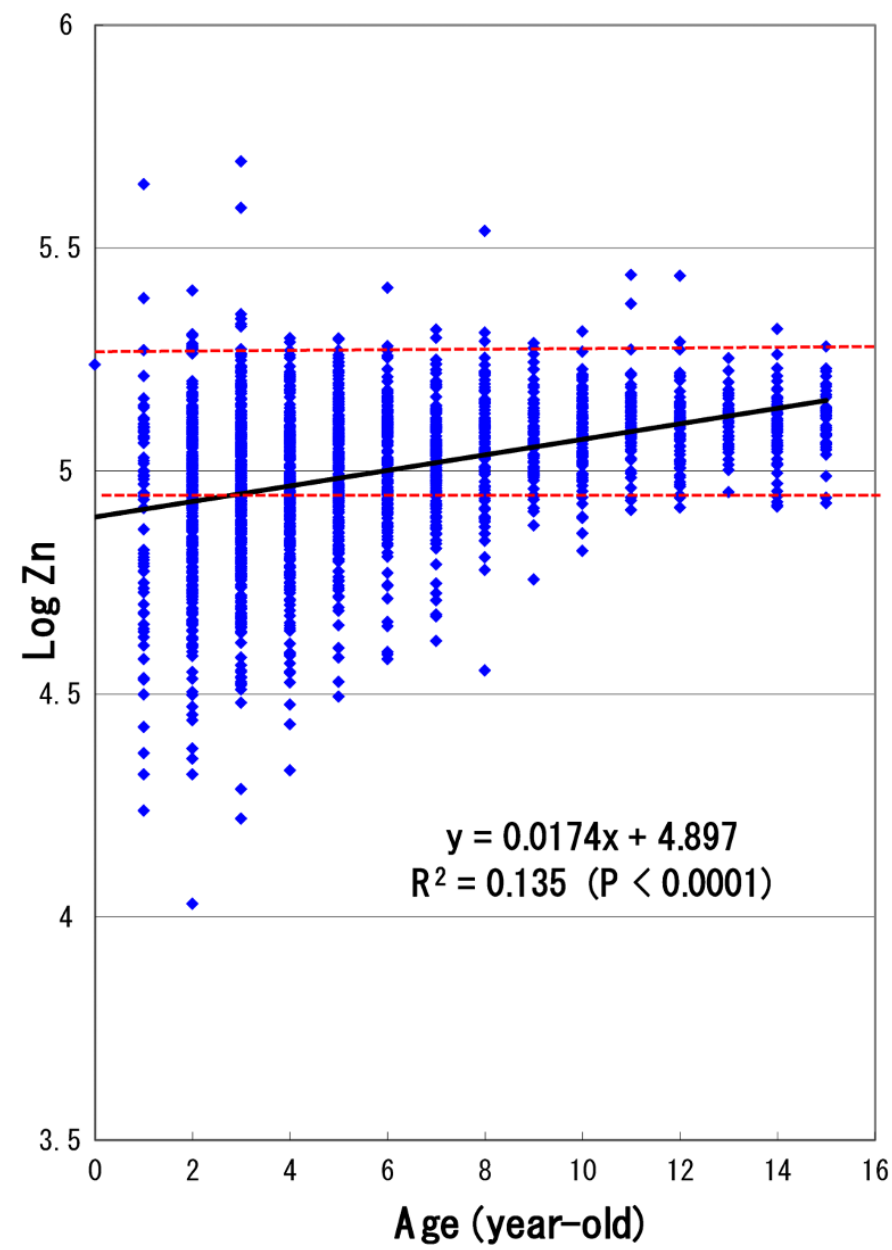

Figure $2 \mid$ Age related increase in zinc concentration in autistic children. The association of hair logarithmic zinc concentration with age in autistic children $(\mathrm{N}=1,967)$ is shown. Each spot represents the corresponding age and logarithmic zinc concentration of the respective child. A significant association of the zinc concentration with age $(\mathrm{r}=0.367, \mathrm{p}<0.0001)$ in the autistic children is shown.
Table 1 | Geometric mean zinc concentrations in autistic children

\begin{tabular}{lccc} 
Gender & Age & No. & Mean Zn Level \\
\hline Male & 0-3 year-old & $\mathrm{N}=577$ & 87 \\
& 4-9 year-old & $\mathrm{N}=736$ & 100 \\
Female & $10-15$ year-old & $\mathrm{N}=240$ & 124 \\
& $0-3$ year-old & $\mathrm{N}=118$ & 81 \\
& 4-9 year-old & $\mathrm{N}=181$ & 104 \\
& $10-15$ year-old & $\mathrm{N}=115$ & 131 \\
\hline
\end{tabular}

The geometric mean zinc concentrations (ppm) in the age groups of 0-3, 4-9 and 10-15 year-old in 1,967 autistic children (1,553 males and 414 females) are tabled.

tailing in lower range, and 584 in 1,967 subjects (29.7\%) were found to have low zinc concentration less than -2 S.D. (standard deviation) level of the control reference range (86.3-193 micro g/g hair (ppm); geometric mean $=129 \mathrm{ppm}$ ), estimated as zinc deficiency. The logarithmic zinc concentrations in the autistic subjects showed a significant correlation with age $(\mathrm{r}=0.367, \mathrm{p}<0.0001)$, with a plateau at over 10 year-old (Fig. 2). The only one 0 year-old case in 1,967 subjects was 11 month-old female with no zinc deficiency (Fig. 2) and may be a suspect, because she was found to suffer from high burdens with aluminium, lead, iron and copper (data not shown). The minimum zinc concentration of $10.7 \mathrm{ppm}$ was observed in a 2-year-old boy, corresponding to about $1 / 12$ of the control mean level. The geometric mean zinc concentration was lowest in the age group of $0-3$ year-old in both sexes (Table 1). The incidence rate of zinc deficiency in the age group of 0-3 and 4-9 year-old was estimated 43.5 and $28.1 \%$ in male, and 52.5 and $28.7 \%$ in female, respectively (Table 2 ). In the group aged $10-15$ year-old, the zinc deficiency rate in male and female was calculated 3.3 and $3.5 \%$, respectively. There was little marked gender difference observed in scalp hair zinc concentration and incidence rate of zinc deficiency in the subjects with autism.

\section{Discussion}

Zinc is an essential trace element that plays important roles in nucleic acid/protein synthesis, cell replication, tissue growth and repair, especially in pregnant women and infants. Therefore, zinc deficiency is known associated with various pathological conditions, including impaired immunity, delayed wound healing, retarded growth, neural development disorders and degenerative diseases ${ }^{23,24}$.

Hair zinc is commonly used in marginal zinc deficiency studies of children, and its usefulness has been documented in many industrialized countries including Canada and the USA ${ }^{25-29}$. In the present study, we determined zinc concentration in scalp hair samples from 1,967 subjects ( 1,553 males and 414 females) diagnosed with autism by physicians, and investigated any association of zinc deficiency with autistic spectrum disorders.

The control zinc reference range for healthy subjects published by ourselves $^{17,18}$ was almost consistent with those reported by other

\begin{tabular}{|lcccc}
\hline Table 2 $\mid$ Incidence rate of zinc deficiency in autistic children \\
Gender & Age & No. & $\begin{array}{c}\text { Number of } \\
\text { Cases with Zn } \\
\text { Deficiency }\end{array}$ & $\begin{array}{c}\text { Rate } \\
\text { (\%) of } \mathrm{Zn} \\
\text { Deficiency }\end{array}$ \\
\hline Male & $0-3$ year-old & $\mathrm{N}=577$ & 251 & 43.5 \\
& $4-9$ year-old & $\mathrm{N}=736$ & 207 & 28.1 \\
& $10-15$ year-old & $\mathrm{N}=240$ & 8 & 3.3 \\
Female & $0-3$ year-old & $\mathrm{N}=118$ & 62 & 52.5 \\
& $4-9$ year-old & $\mathrm{N}=181$ & 52 & 28.7 \\
& $10-15$ year-old & $\mathrm{N}=115$ & 4 & 3.5 \\
\hline
\end{tabular}

The number and incidence rate of the individuals with zinc deficiency in 1,967 autistic children (1,553 males and 414 females) are tabled. 
research groups ${ }^{10-12}$ and those for nutritionally-controlled children reported by Munakata et al. ${ }^{13}$ and Vancouver preschoolers aged $2-5$ year-old $(\mathrm{N}=719)$ by Vaghri et al. ${ }^{28,29}$. Based on these findings, the normal human hair zinc level was estimated to be around $130 \mathrm{ppm}$ independently age and sex, although it was relatively low during childhood ${ }^{19,28}$ (Fig. 2) and infants seem liable to fall into zinc deficiency because of their higher necessity of zinc for developing and growing. Thus, the lower zinc levels for control children reported by Adams et al. ${ }^{15}$, Munakata et al. ${ }^{13}$ and ourselves ${ }^{14}$ should be re-considered as non-normal control values including nonhealthy, affected zinc levels, as Adams themselves recently reviewed that the $11 \%$ participants in the neurotypical control children group were medicated with drugs such as anti-asthma/allergies or anti-incontinence ${ }^{30}$.

The histogram of logarithmic hair zinc concentration in the 1,967 autistic children showed a non-symmetric profile with a marked tailing in lower range, indicating that about $30 \%$ of the subjects with autism have low zinc concentrations less than -2 S.D. level $(<86.3 \mathrm{ppm})$ of the control reference range, being estimated as marginal zinc deficiency (Fig. 1). In particular, nearly one half (male: $43.5 \%$; female: $52.5 \%$ ) of the autistic infants aged $0-3$ year-old were found to suffer from marginal to severe zinc deficiency (Table 2).

A significant correlation $(\mathrm{r}=0.367, \mathrm{p}<0.0001)$ of the zinc concentration with age in the autistic subjects (Fig. 2) suggests that children, in particular infants, need larger amount of zinc (per kg body weight) for their development and growth and so they are liable to zinc deficiency. In fact, the minimum zinc concentration as low as $10.7 \mathrm{ppm}$ corresponding to about $1 / 12$ of the control level was observed in a 2-year-old boy (Fig. 2), and the lowest mean concentration level was observed in the infant group aged 0-3 year-old in both sexes (male: $87 \mathrm{ppm}$; female: $81 \mathrm{ppm}$ ) (Table 1). These findings indicate that a considerable number of autistic children, in particular infants, are suffered from marginal to severe zinc deficiency, suggesting that infantile zinc deficiency may play a role in the neural development disorders and pathogenesis of autism spectrum disorders, and zinc supplementation may be useful for treatment and prevention of some patients with autistic disorders and suspects.

The incidence rate of zinc deficiency in the age group of 4-9 and 10-15 year-old was estimated 28.1 and $3.3 \%$ in male, and 28.7 and $3.5 \%$ in female subjects, respectively, indicating that there is little gender difference in zinc deficiency rate in the autistic subjects. It remains to be explained why the elder subjects over 10-year-old have normal zinc concentration, although this is maybe explained by the possibility that the young adolescents have better metal-protein functioning and utilize dietary zinc more efficiently and effectively.

There were some studies reporting lower serum zinc levels in children with attention deficit hyperactivity disorder (ADHD) in developing countries ${ }^{24,31}$. Recently, zinc deficiency has been remarked in not only developing but also developed countries, due to imbalanced intake of trace nutrients and dieting ${ }^{32,33}$. Faber et al. ${ }^{34}$ reported that the mean plasma $\mathrm{Zn} / \mathrm{Cu}$ ratio in children with autistic disorder was below the cut-off value of the lowest $2.5 \%$ of healthy children, suggesting that plasma zinc/copper ratio may be a biomarker of heavy metal toxicity in children with autism spectrum disorders. Other clinical studies reported that mean serum zinc levels were significantly lower in both autism and ADHD groups, and that serum zinc level correlated inversely with parent- and teacher-rated inattention in ADHD children ${ }^{33}$. Furthermore, zinc treatment was reported significantly superior to placebo in reducing symptoms of hyperactivity, impulsivity and impaired socialization in ADHD patients ${ }^{35,36}$. The other human study showed that many children with ADHD have lower zinc concentration in comparison to healthy children, and zinc supplement as an adjunct to methylphenidate has favourable effects in the treatment of ADHD children, pointing to the possible association of zinc deficiency and ADHD pathophysiology ${ }^{37}$.
The cellular zinc homeostasis is mediated by two transporter families: the zinc-importer (Zip) family that transport zinc into the cytosol, and the zinc transporter ( $\mathrm{ZnT}$ ) family transporting zinc out of the cytosol $^{38}$. The severe zinc deficiency in a rare inherited human disease "acrodermatitis enteropathica" is reported to result from defective intestinal absorption of zinc by mutations in the Zip4 transporter located on intestinal duct ${ }^{39,40}$. Autistic children have often been reported to have gastrointestinal problems of intestinal mal-absorption of nutrients ${ }^{41}$, named "leaky gut syndrome" which may lead to zinc deficiency, and zinc deficiency itself may further lead to gut syndrome resulting from over-consumption of fructose ${ }^{42}$. Furthermore, many of the autistic children are also suffered from allergic disorders that are known to relate to zinc deficiency. Thus, these findings suggest that infantile zinc deficiency may be also responsible for the pathogenesis of various symptoms observed in the children with autistic spectrum disorders.

Recently, dietary restriction-induced zinc deficiency has been reported to up-regulate intestinal zinc-importer (Zip4) and induce the increase in Zip4 protein located to the plasma membrane of enterocytes ${ }^{43}$. This adoptive response to zinc deficiency is known to lead to increasing in the risk of high-uptake of toxic metals such as cadmium and lead. Thus, infants with zinc deficiency are liable to be exposed to increased risk of absorbing high amount of toxic metals and retaining them in their body. These findings suggest that the increased risk of toxic metal burdens attendant on the infantile zinc deficiency may also contribute to the pathogenesis of autistic spectrum disorders.

The mechanisms of zinc deficiency in autistic infants and ADHD children may be explained by their unbalanced nutritional intake and lower absorption ability in intestinal duct ${ }^{41,44}$. In addition, maternal cigarette smoking has been reported to be associated with lower zinc and higher cadmium and lead concentrations in their neonates ${ }^{45}$. Because of their high distribution property to bone tissue, during pregnancy and lactation, these toxic metals accumulated in the maternal bone matrix are co-transferred with calcium and magnesium to foetal and newborn bodies through increased bone-resorption ${ }^{45-47}$.

Kern et al. ${ }^{48}$ reported that arsenic, cadmium and lead were significantly lower in the hair of children with autism than in matched controls, and the patients have trouble excreting these toxic elements. We also reported that not only toxic but also some essential metals are significantly lower in autistic children ${ }^{13}$, suggesting that the patients with zinc deficiency have a low excretion ability of toxic metals and result in a higher body burden with them. Eklund and Oskarsson $^{49}$ reported that soy-based formulas contain approximately six times more cadmium than cow's milk formulas, and cereal-based formulas have 4-21 times higher levels. Thus, the dietary intake of cadmium may be higher in the children feeding on infant formulas and weaning foods, compared to breast milk-fed infants.

In conclusion, this preliminary study shows that many of infants with autistic spectrum disorders have been suffered from marginal to severe zinc deficiency. This finding suggests that infantile zinc deficiency and attendant toxic metal accumulation may play principal roles in the pathogenesis of autism, in particular, through their epigenetic modifications. Furthermore, these findings suggest the possibility that some of autistic children and suspects may be improved by nutritional approach supplementing deficient nutrients such as zinc, on the basis of evidence data, although zinc supplementation should be applied for infantile patients rather than elder group over 10 -year-old. This evidence-based nutritional approach may yield a novel pathway into prevention and treatment of the children with autism spectrum disorders in near future. Controlled studies for this nutritional approach are desirable to establish the principal roles of infantile zinc deficiency and toxic metal burdens in the neural development disorders and pathogenesis of autism. 
17. Yasuda, H., Yonashiro, T., Yoshida, K., Ishii, T. \& Tsutsui, T. Relationship between body mass index and minerals in male Japanese adults. Biomed. Res. Trace Elem. 17, 316-321 (2006).

Sampling and zinc analysis. On the basis of informed consent, scalp hair samples from 1,967 (male: 1,553; female: 414 ) subjects aged $0-15$ years were collected in the period from June 2005 to September 2007, although 0 year-old subject was only one (11 month-old female). These subjects were comprised of the children diagnosed with autistic spectrum disorders by their physicians. Hair sampling was recommended to cut as close to the scalp of the occipital area as possible.

Hair sample of $75 \mathrm{mg}$ was weighed into $50 \mathrm{ml}$ plastic tube, and washed with acetone and then with $0.01 \%$ Triton solution, in accordance with the procedures recommended by the Hair Analysis Standardization Board. The washed hair sample was mixed with $10 \mathrm{ml}$ 6.25\% tetra methyl ammonium hydroxide (TMAH, Tama Chemical, Kawasaki, Japan) and 50 micro-L $0.1 \%$ gold solution (SPEX Certi Prep, Metuchen, NJ, USA), and then dissolved at $75{ }^{\circ} \mathrm{C}$ with shaking for 2 hours. After cooling the solution to room temperature, internal standard solution was added, and after adjusting its volume gravimetric, the obtained solution was used for zinc analysis. The zinc concentrations were determined with inductively coupled plasma mass spectrometry (ICP-MS; 7500ce, Agilent Technologies, Santa Clara, CA, USA) by the internal standard method and expressed as ng/g hair (ppb) or micro-g/g (ppm) ${ }^{17,18}$. The method detection limit for zinc determination was $12 \mathrm{ng} / \mathrm{g}$ hair (ppb), and human hair certified reference material (NIES CRM No. 13) was used to check for the accuracy of analysis. The inter-daily variation of zinc determination was calculated $2.2 \%$. The control zinc reference range of $86.3-193 \mathrm{ppm}$, its geometric mean \pm 2 S.D. level, obtained from the data for healthy 436 male subjects aged 21-40 year-old was close to that for healthy female adults, as previously reported ${ }^{17,18,50}$, furthermore almost consisting with those reported by the other research groups ${ }^{10-12}$. This control zinc reference range was also almost consistent with those for nutritionally controlled children ${ }^{13}$ and for the Vancouver preschoolers aged 2-5 year-old $(\mathrm{N}=719)^{28}$, but somewhat different from the age-matched reference data for neurotypical control children in which the $11 \%$ participants had been medicated as reviewed by Adams ${ }^{30}$.

This study has been approved by review of the ethical committee of La Belle Vie research laboratory. All of the data obtained are held securely in such a form as to ensure anonymity.

Statistical analysis. Because scalp hair mineral concentration is log-normally distributed, the zinc concentration was converted to logarithm, and the geometric rather than arithmetic mean was used as representative of its hair concentration. The relation between age and zinc concentrations of the subjects was examined by Pearson's correlation coefficient test.

1. Pinto, D. et al. Functional impact of global rare copy number variation in autism spectrum disorders. Nature 466, 368-372 (2010).

2. Chakrabarti, S. \& Fombonne, E. Pervasive developmental disorders in preschool children: confirmation of high prevalence. Am. J. Psychiatry 162, 1133-1141 (2005).

3. Bailey, A. et al. Autism as a strongly genetic disorder: evidence from a British twin study. Psychol. Med. 25, 63-77 (1995).

4. Marshall, C. R. et al. Structural variation of chromosomes in autism spectrum disorder. Am. J. Hum. Genet. 82, 477-488 (2008).

5. Dufault, R. et al. Mercury exposure, nutritional deficiencies and metabolic disruptions may affect learning in children. Behav. Brain Funct. 5, 44-58 (2009).

6. O'Rahilly, S. Human genetics illuminates the paths to metabolic disease. Nature 462, 307-314 (2009).

7. Jin, Y. H. et al. Cadmium is a mutagen that acts by inhibiting mismatch repair. Nat. Genet. 34, 239-241 (2003).

8. Takiguchi, M., Achanzar, W. E., Qu, W., Li, G. \& Waalkes, M. P. Effects of cadmium on DNA-(cytosine-5) methyltransferase activity and DNA methylation status during cadmium-induced cellular transformation. Exp. Cell Res. 286, 355365 (2003).

9. Arita, A. \& Costa, M. Epigenetics in metal carcinogenesis: nickel, arsenic, chromium and cadmium. Metallomics 1, 222-228 (2009).

10. Rodushkin, I. \& Axelsson, M. D. Application of double focusing sector field ICPMS for multi elemental characterization of human hair and nails. Part 1. Analytical methodology. Sci. Total Environ. 250, 83-100 (2000).

11. Goulle, J. P. et al. Metal and metalloid multi-elementary ICP-MS validation in whole blood, plasma, urine and hair: Reference values. Forens. Sci. Intern. 153, 3944 (2005).

12. Wang, C. T., Chang, W. T., Zeng, W. F. \& Lin, C. H. Concentrations of calcium, copper, iron, magnesium, potassium, sodium and zinc in adult female hair with different body mass indexes in Taiwan. Clin. Chem. Lab. Med. 43, 389-393 (2005).

13. Munakata, M. et al. A preliminary analysis of trace elements in the scalp hair of patients with severe motor disabilities receiving enteral nutrition. Brain Development 28, 521-525 (2006).

14. Yasuda, H., Yonashiro, T., Yoshida, K., Ishii, T. \& Tsutsui, T. Mineral imbalance in children with autistic disorders. Biomed. Res. Trace Elem. 16, 285-291 (2005).

15. Adams, J. B., Holloway, C. E., George, F. \& Quig, D. Analyses of toxic metals and essential minerals in the hair of Arizona children with autism and associated conditions, and their mothers. Biol. Trace Elem. Res. 110, 193-209 (2006).

16. Adams, J. B. et al. The severity of autism is associated with toxic metal body burden and red blood cell glutathione levels. J. Toxicol. 2009, 532640 (2009).
18. Yasuda, H. et al. Metallomics study using hair mineral analysis and multiple logistic regression analysis: relationship between cancer and minerals. Environ. Health Prev. Med. 14, 261-266 (2009).

19. Pilch, S. M. \& Senti, F. R. Analysis of zinc data from the second national health and nutrition examination survey (NHANES II). J. Nutr. 115, 1393-1397 (1985).

20. Yasuda, H., Yonashiro, T., Yoshida, K., Ishii, T. \& Tsutsui, T. High toxic metal levels in scalp hair of infants and children. Biomed. Res. Trace Elem. 16, 39-45 (2005).

21. Yasuda, H., Yoshida, K., Segawa, M., Tokuda, R., Yasuda, Y. \& Tsutsui, T. High accumulation of aluminium in hairs of infants and children. Biomed. Res. Trace Elem. 19, 57-62 (2008)

22. Yasuda, H., Yoshida, K., Yasuda, Y. \& Tsutsui, T. Two age-related accumulation profiles of toxic metals. Cur. Aging Sci. 4, (2011) (in press) PMID: 21834789.

23. Prasad, A. S. Impact of the discovery of human zinc deficiency on health. J. Am. Coll. Nutr. 28, 257-265 (2009).

24. DiGirolamo, A. M. \& Raminez-Zea, M. Role of zinc in maternal and child mental health. Am. J. Clin. Nutr. 89(suppl), 940S-945S (2009).

25. Gibson, R. S., Vanderkooy, P. D., MacDonald, A. C., Goldman, A., Ryan, B. A. \& Berry, M. A. Growth-limiting, mild zinc-deficiency syndrome in some southern Ontario boys with low height percentiles. Am. J. Clin. Nutr. 49, 1266-1273 (1989).

26. Walravens, P. A., Krebs, N. F. \& Hambidge, K. M. Linear growth of low income preschool children receiving a zinc supplement. Am. J. Clin. Nutr. 38, 195-201 (1983).

27. Gibson, R. S. Assessment of trace element status in humans. Prog. Food Nutr. Sci. 13, 67-111 (1989).

28. Vaghri, Z., Barr, S., Wong, H., Chapman, G. \& Hertzman, C. Age-based differences in hair zinc of Vancouver preschoolers. Biol. Trace Elem. Res. 126, S21-S30 (2008).

29. Vaghri, Z., Wong, H., Barr, S., Chapman, G. \& Hertzman, C. Associations of socio-demographic and behavioural variables with hair zinc of Vancouver preschoolers. Biol. Trace Elem. Res. 129, (2011) PMID: 21369714.

30. Adams, J. B. et al. Nutritional and metabolic status of children with autism vs. neurotypical children, and the association with autism severity. Nutr. Metab. 8 , 34-65 (2011).

31. Arnold, L. E. \& DiSilvestro, R. A. Zinc in attention-deficit/hyperactivity disorder. J. Child Adolesc. Psychopharmacol. 15, 619-627 (2005).

32. Plum, L. M., Rink, L. \& Haase, H. The essential toxin: Impact of zinc on human health. Int. J. Environ. Res. Public Health 7, 1342-1365 (2010).

33. Arnold, L. E. et al. Serum zinc correlates with parent- and teacher-rated inattention in children with attention-deficit/hyperactivity disorder. J. Child Adolesc. Psychopharmacol. 15, 628-636 (2005).

34. Faber, S., Zinn, G. M., Faber, S., Zinn, G. M., Kern, J. C. \& Kingston, H. M. The plasma zinc/serum copper ratio as a biomarker in children with autism spectrum disorders. Biomarkers 14, 171-180 (2009)

35. Yorbik, O., Ozdag, M. F., Olgun, A., Senol, M. G., Bek, S. \& Akman, S. Potential effects of zinc on information processing in boys with attention deficit hyperactivity disorder. Prog. Neuropsychopharmacol. Biol. Psychiatry 32, 662-667 (2008)

36. DiGirolamo, A. M. et al. Randomized trial of the effect of zinc supplementation on the mental health of school-age children in Guatemala. Am. J. Clin. Nutr. 92, 1241-1250 (2010)

37. Akhondzadeh, S., Mohammadi, M. R. \& Khademi, M. Zinc sulfate as an adjunct to methylphenidate for the treatment of attention deficit hyperactivity disorder in children: a double blind and randomised trial [ISRCTN64132371]. B.M.C. Psychiatry 4, 9-14 (2004).

38. Lichten, L. A. \& Cousins, R. J. Mammalian zinc transporters: nutritional and physiologic regulation. Ann. Rev. Nutr. 29, 153-176 (2009).

39. Wang, K., Zhou, B., Kuo, Y., Zemansky, J. \& Gitschier, J. A novel member of a zinc transporter family is defective in acrodermatitis enteropathica. Am. J. Hum. Genet. 71, 66-73 (2002)

40. Dufner-Beattie, J., Wang, F., Kuo, Y. M., Gitschier, J., Eide, D. \& Andrews, G. K. The acrodermatitis enteropathica gene ZIP4 encodes a tissue-specific, zincregulated zinc transporter in mice. J. Biol. Chem. 278, 33474-33481 (2003).

41. Adams, J. B., Johansen, L. J., Powell, L. D., Quig, D. \& Rubin, R. A. Gastrointestinal flora and gastrointestinal status in children with autism - comparisons to typical children and correlation with autism severity. BMC Gastroenterology 11, 22-34 (2011).

42. Lustig, R. H. Fructose: metabolic, hedonic, and social parallels with ethanol. J. Am. Diet. Assoc. 110, 1307-1321 (2010).

43. Cousins, R. J., Liuzzi, J. P. \& Lichten, L. A. Mammalian zinc transport, trafficking, and signals. J. Biol. Chem. 281, 24085-24089 (2006).

44. Dodig-Curkovic, K., Dovhanj, J., Curković, M., Dodig-Radić, J. \& Degmecić, D. The role of zinc in the treatment of hyperactivity disorder in children. Acta Med. Croatica 63, 307-313 (2009).

45. Razagui, I. B. \& Ghribi, I. Maternal and neonatal scalp hair concentrations of zinc, cadmium, and lead: relationship to some lifestyle factors. Biol. Trace Elem. Res. 106, 1-28 (2005).

46. Symanski, E. \& Hertz-Picciotto, I. Blood lead levels in relation to menopause, smoking, and pregnancy history. Am. J. Epidemiol. 141, 1047-1058 (1995). 
47. Gulson, B. L., Jameson, C. W., Mahaffey, K. R., Mizon, K. J., Korsch, M. J. \& Vimpani, G. Pregnancy increases mobilization of lead from maternal skeleton. J. Lab. Clin. Med. 130, 51-62 (1997).

48. Kern, J. K., Granneman, B. D., Trivedi, M. H. \& Adams, J. B. Sulfhydryl-reactive metals in autism. J. Toxicol. Environm. Health 70, 715-721 (2007).

49. Eklund, G. \& Oskarsson, A. Exposure of cadmium from infant formulas and weaning foods. Food Addit. Contam. 16, 509-519 (1999).

50. Yasuda, H., Yoshida, K., Fukuchi, K., Tokuda, R., Tsutsui, T. \& Yonei, Y. Association of aging with minerals in Japanese male adults. Anti-Aging Med. 4, 38-42 (2007).

\section{Acknowledgements}

The authors thank the subjects and their parents for collaboration to this study.

\section{Author contributions}

H.Y. performed this study, analysed the data and wrote the manuscript with the help of K.Y., Y.Y. and T.T. K.Y. performed mass spectrometry analysis, and T.T. supervised this study. All authors discussed the results and commented on the manuscript.

\section{Additional information}

Competing financial interests: The authors declare that they have no competing financial interests.

License: This work is licensed under a Creative Commons

Attribution-NonCommercial-ShareAlike 3.0 Unported License. To view a copy of this license, visit http://creativecommons.org/licenses/by-nc-sa/3.0/

How to cite this article: Yasuda, H., Yoshida, K., Yasuda, Y. \& Tsutsui, T. Infantile zinc deficiency: Association with autism spectrum disorders. Sci. Rep. 1, 129; DOI:10.1038/ srep00129 (2011). 